\title{
TU/e emonown

\section{Implementation of the nodal discontinuous Galerkin method for the plate vibration problem using linear elasticity equations}

Citation for published version (APA):

Sihar, I., \& Hornikx, M. (2019). Implementation of the nodal discontinuous Galerkin method for the plate vibration problem using linear elasticity equations. Acta Acustica united with Acustica, 105(4), 668-681.

https://doi.org/10.3813/AAA.919347

DOI:

10.3813/AAA.919347

Document status and date:

Published: 01/07/2019

\section{Document Version:}

Publisher's PDF, also known as Version of Record (includes final page, issue and volume numbers)

\section{Please check the document version of this publication:}

- A submitted manuscript is the version of the article upon submission and before peer-review. There can be important differences between the submitted version and the official published version of record. People interested in the research are advised to contact the author for the final version of the publication, or visit the $\mathrm{DOI}$ to the publisher's website.

- The final author version and the galley proof are versions of the publication after peer review.

- The final published version features the final layout of the paper including the volume, issue and page numbers.

Link to publication

\section{General rights}

Copyright and moral rights for the publications made accessible in the public portal are retained by the authors and/or other copyright owners and it is a condition of accessing publications that users recognise and abide by the legal requirements associated with these rights.

- Users may download and print one copy of any publication from the public portal for the purpose of private study or research.

- You may not further distribute the material or use it for any profit-making activity or commercial gain

- You may freely distribute the URL identifying the publication in the public portal.

If the publication is distributed under the terms of Article 25fa of the Dutch Copyright Act, indicated by the "Taverne" license above, please follow below link for the End User Agreement:

www.tue.nl/taverne

Take down policy

If you believe that this document breaches copyright please contact us at:

openaccess@tue.nl

providing details and we will investigate your claim. 


\title{
Implementation of the Nodal Discontinuous Galerkin Method for the Plate Vibration Problem using Linear Elasticity Equations
}

\author{
Indra Sihar, Maarten Hornikx \\ Eindhoven University of Technology, Eindhoven, The Netherlands. [i.sihar, m.c.j.hornikx]@tue.nl
}

\begin{abstract}
Summary
This work presents a numerical solution of the forced plate vibration problem using the nodal discontinuous Galerkin (DG) method. The plate is modelled as a three-dimensional domain, and its vibration is governed by the linear elasticity equations. The nodal DG method discretises the spatial domain and computes the spatial derivatives of the equations element-wise, while the time integration is conducted using the Runge-Kutta method. This method is in particular of interest as it is very favourable to carry out the computation by a parallel implementation. Several aspects regarding the numerical implementation such as the plate boundary conditions, the point force excitation, and the upwind numerical flux are presented. The numerical results are validated for rectangular concrete plates with different sets of boundary conditions and thicknesses, by a comparison with the exact mobilities that are derived from the classical plate theory (CPT) and the first order shear deformation theory (FSDT). The plate thickness is varied to understand its effect regarding the comparison with the CPT. An excellent agreement between the numerical solution and the FSDT was found. The agreement with the CPT occurs only at the first couple of resonance frequencies, and as the plate is getting thinner. Furthermore, the numerical example is extended to an L-shaped concrete plate. The mobility is then compared with the mobilities obtained by the CPT, FSDT, and linear elasticity equations.
\end{abstract}

PACS no. 43.20.Ks, 43.40.Dx, 43.55.Ka, 43.55.Rg

\section{Introduction}

To obtain a good building acoustic performance, an accurate prediction of the vibro-acoustic characteristics of the building components is essential. In general, building components, such as floors and walls, are modelled as monolithic structures to obtain its vibro-acoustic characteristics. However, when a building component involves multiple structures such as beams, plates, and cavities, the wave dynamics are complex, and the monolithic models are not sufficient to represent the structures. Moreover, when waves propagate across junctions or complex structures (i.e. non-homogeneous walls), a more general model is needed to predict the vibro-acoustic characteristics. To solve this problem, one may use the energy flow model such as presented in the European standard EN 12354, or a more general statistical energy analysis [1]. However, these methods are based on the assumption that the sound and vibration fields are diffuse and have sufficient modal counts in each sub-system, while in the low-frequency range this assumption is no longer appropriate. In that case, a wave-based method that capable to couple the dynamics of the air and the structure is of interest to predict the vibro-acoustic characteristics.

Received 13 June 2018

accepted 7 May 2019.
One approach is by modelling both the structural vibration and the wave propagation in air using the linear elasticity (LE) equations [2, 3]. The LE equations are often used in solid mechanics studies, yet its implementation to the vibro-acoustic problem is also feasible. There are advantages of using the LE equations to solve vibro-acoustic problems in a building. One advantage is when the thin structure assumption does not hold, as thick structures can be solved in the LE equations. Another advantage is when there are changes in the cross-section area due to stiffeners, junctions, or any discontinuities, as they could be treated directly in the model.

In the geophysics field, the LE equations have frequently been used to model seismic waves propagation. For instance, Vireux used the 2-D LE equations to model the Rayleigh's surface waves and solved it using the finite difference time-domain (FDTD) method [4]. Dumbser and Käser modelled the 3-D seismic wave propagation using LE equations and solved it using the arbitrary high-order derivatives discontinuous Galerkin (ADER-DG) method [5]. Another example is by Wilcox et al., who used DG for to simulate the seismic waves propagation through coupled acoustic-elastic media [6].

In the field of sound and vibration, Toyoda and Takahashi [7] have used the LE equations to model the impact sound in a two-story building and solved it using the FDTD method. Another example is by Xuan et al. who ap- 
plied the LE equations to the structural-acoustic problem and solved it using the finite volume method (FVM) [8]. Additionally, Bermúdez et al. have modelled the vibroacoustic problem of a 3-D vessel filled with fluid using LE equations and used the finite element method (FEM) to calculate its eigenvalues [9].

In solving a real-life building acoustics problem, there is a need for a more efficient method to solve the LE equations than FDTD, FVM, and FEM. This efficiency can be provided by the discontinuous Galerkin (DG) method. This method is in particular of interest as it is very favourable to carry out DG calculations by a parallel implementation, opening opportunities to solve real-world problems in a reasonable computation time.

In this paper, as a first step to solve the vibro-acoustic problem in a complex building structure, the forced vibration of plates are modelled. The vibration is modelled using the LE equations and then solved numerically using the nodal DG method. The nodal DG method satisfies the strong form of LE equations by utilising the Lagrange interpolation polynomials as the basis functions, and the Runge-Kutta method as the time integration solver. It has been used widely to solve the electromagnetic and fluid mechanics problems [10]. Furthermore, the hybrid approach of the nodal DG method and Fourier pseudospectral time-domain has been developed by Pagán Muñoz and Hornikx to solve the acoustic propagation problem in air [11], which can be coupled with the current vibration problem.

The remainder of this paper is organised as follows. Section 2 introduces the LE equations. In Section 3, the numerical aspects such as the nodal DG formulation, the upwind numerical flux, and the point force excitation are presented. Section 4 introduces rectangular plates and Lshaped plate configurations. In this section, the problem domains, boundary conditions (BCs), and numerical settings are given. Finally, in Section 5, the comparison of transfer mobilities between numerical and exact solutions are discussed.

\section{Linear elasticity equations}

In solid media, the structural vibration can be modelled using the equations of motion and the constitutive equations. This set of equations is known as the linear elasticity equations. The LE equations for isotropic structures can be written in the stress-velocity form using 3-D rectangular Cartesian coordinates as

$$
\begin{aligned}
& \frac{\partial \mathbf{q}}{\partial t}+\nabla \cdot(\mathbf{A q})=\mathbf{g}, \\
& \mathbf{q}=\left[\begin{array}{lllllllll}
v_{x} & v_{y} & v_{z} & \tau_{x x} & \tau_{y y} & \tau_{z z} & \tau_{x z} & \tau_{y z} & \tau_{x y}
\end{array}\right]^{T}, \\
& \mathbf{g}=\left[\begin{array}{lllllllll}
g_{x} & g_{y} & g_{z} & 0 & 0 & 0 & 0 & 0 & 0
\end{array}\right]^{T}, \\
& \mathbf{A}=\left[\begin{array}{c|c}
\mathbf{0}_{3 \times 3} & \mathbf{A}_{\mathbf{1}} \\
\hline \mathbf{A}_{2} & \mathbf{0}_{6 \times 6}
\end{array}\right], \\
& \mathbf{A}_{\mathbf{1}}=-\frac{1}{\rho}\left[\begin{array}{cccccc}
n_{x} & 0 & 0 & n_{z} & 0 & n_{y} \\
0 & n_{y} & 0 & 0 & n_{z} & n_{x} \\
0 & 0 & n_{z} & n_{x} & n_{y} & 0
\end{array}\right] \text {, }
\end{aligned}
$$

where $\varphi_{i}(\boldsymbol{x})$ are the 3-D Lagrange interpolation polynomials based on nodal points $\boldsymbol{x}_{i}, N_{p}$ is the number of the nodal points, $\Psi_{j}(\boldsymbol{x})$ are 3-D orthogonal basis functions, and $\hat{\mathbf{q}}_{j}^{k}$ are the coefficients of the basis functions. The expression of $\Psi_{j}(\boldsymbol{x})$ is given in Section 3.3.

The first term of Equation (2) is known as the nodal form, and the second term is known as the modal form. In the nodal DG method, the nodal form is used to approximate the solution. However, the closed-form expression of the Lagrange interpolation polynomials in tetrahedral elements does not exist; therefore it is constructed by utilising the modal form. The relationship between nodal and modal form is established by the Vandermonde matrix (V) as

$\mathbf{q}=\mathbf{V} \hat{\mathbf{q}}, \quad \mathrm{V}_{i j}=\Psi_{j}\left(x_{i}\right), \quad \boldsymbol{\varphi}(\boldsymbol{x})=\left(\mathbf{V}^{T}\right)^{-1} \boldsymbol{\Psi}(\boldsymbol{x})$

In each element, we need to have a minimum number of nodal points $N_{p}$ to establish Lagrange interpolation polynomials of order $N . N_{p}$ is defined as $N_{p}=(N+1)(N+$ 
2) $(N+3) / 6$. Moreover, to have a well-conditioned Vandermonde matrix, the nodal points are distributed following the optimised Legendre Gauss Lobato (LGL) points over a tetrahedral element [10].

Afterwards, Equation (2) is substituted into Equation (1), and multiplied by the test function following the Galerkin approach. By conducting spatial integration by parts twice, we arrive at the problem's strong formulation at each element as

$$
\begin{aligned}
& \int_{D_{k}}\left[\frac{\partial \mathbf{q}_{h}^{k}}{\partial t}+\nabla \cdot\left(\mathbf{A} \mathbf{q}_{h}^{k}\right)\right] \varphi_{i} \mathrm{~d} \boldsymbol{x}= \\
& \int_{D_{k}} \mathbf{g}_{h}^{k} \varphi_{i} \mathrm{~d} \boldsymbol{x}-\int_{\partial D_{k}} \mathbf{n} \cdot\left[\mathbf{f}^{*}-\mathbf{A} \mathbf{q}_{h}^{k}\right] \varphi_{i} \mathrm{~d} \boldsymbol{x},
\end{aligned}
$$

where $\partial D_{k}$ is the element surface, $\mathbf{g}_{h}^{k}$ is the approximated body force vector, and $\mathbf{f}^{*}$ is the numerical flux.

Equation (4) can be solved element-wise, and the numerical flux has a role in ensuring the continuity of the global solution. This role makes the selection of numerical flux is vital in the DG method. In this work, we select the upwind numerical flux as the numerical flux. The details on this flux are elaborated in Section (3.2).

After defining the numerical flux, we substitute the nodal basis and the numerical flux into equation (4) to obtain the semi-discrete form for each element as

$$
\mathbf{M}^{D_{k}} \frac{\partial \mathbf{q}_{h}^{k}}{\partial t}+\sum_{l=x, y, z} \mathbf{K}_{l}^{D_{k}} \mathbf{q}_{h}^{k}=\mathbf{M}^{D_{k}} \mathbf{g}_{h}^{k}-\sum_{m=1}^{4} \mathbf{M}^{\partial D_{k}^{m}} \mathbf{F}^{\partial D_{k}^{m}},
$$

where $\mathbf{M}^{D_{k}}$ is the mass matrix, $\mathbf{K}_{l}^{D_{k}}$ is the stiffness matrix of each spatial derivative $l, \mathbf{M}^{\partial D_{k}^{m}}$ is the mass matrix of the element face $m$ with total four faces on the tetrahedral element, and the $\mathbf{F}^{\partial D_{k}^{m}}$ is the flux term related to the term $\left(\mathbf{f}^{*}-\mathbf{A} \mathbf{q}_{h}^{k}\right)$. For the details on the mass and stiffness matrices, the interested reader is referred to [10]. Having the semi-discrete form at hand, Equation (5) for the whole computational domain can be expressed in the form of ordinary differential equations as

$$
\frac{\mathrm{d} \mathbf{q}}{\mathrm{d} t}=\mathbf{L}(\mathbf{q}(t), t),
$$

where $\mathbf{L}$ is the semi-discrete operator. Finally, various methods could be used to conduct the time integration of Equation (6). In this work, the Runge-Kutta method of fourth order with eight stages (RKF84) by Toulorge is used [13]. In this work, the time step $(\Delta t)$ is defined as

$$
\Delta t=\frac{C_{C F L} \cdot \min \left(r_{D^{k}}\right)}{N^{2} \cdot c_{p}},
$$

where $C_{C F L}$ is the Courant number, $c_{p}$ is the medium longitudinal wave speed, and $r_{D^{k}}$ is the shortest element edge in the computational domain. In the implementation of nodal DG method, the mapping between the standard tetrahedral element and the general tetrahedral element (I) is introduced to generalise the computation. The mapping between $D^{k}$ and $\mathbf{I}$ will be discussed more in Section 3.3.

\subsection{Upwind numerical flux}

In this section, the upwind numerical flux is derived. For more details on the numerical flux, we refer to the work of LeVeque [12], Wilcox et al. [6], or Dumbser et al. [5]. To formulate the upwind numerical flux, the Riemann problem of Equation (1) at the interfaces of each element should be solved. The Riemann problem is a discontinuous initial value problem, where the initial value $(\stackrel{\mathbf{q}}{)})$ is defined as

$$
\stackrel{\mathbf{q}}{ }(x)= \begin{cases}\mathbf{q}^{-} & \text {for } x<0, \\ \mathbf{q}^{+} & \text {for } x>0,\end{cases}
$$

where $\mathbf{q}^{-}$represents the variable at the local element, and $\mathbf{q}^{+}$represents the variable at the neighbor elements. Since Equations (1) are hyperbolic equations, $\mathbf{A}$ can be decomposed as $\mathbf{A}=\mathbf{R} \mathbf{\Lambda} \mathbf{R}^{\mathbf{- 1}}$, with $\boldsymbol{\Lambda}$ the eigen-values, and $\mathbf{R}$ the eigen-vectors. $\boldsymbol{\Lambda}$ can be written as

$$
\begin{aligned}
\Lambda & =\operatorname{diag}\left(-c_{p},-c_{s},-c_{s}, 0,0,0, c_{s}, c_{s}, c_{p}\right), \\
c_{p} & =\sqrt{(\lambda+2 \mu) / \rho}, \quad c_{s}=\sqrt{\mu / \rho},
\end{aligned}
$$

where $c_{p}$ is the longitudinal wave speed, and $c_{s}$ is the shear wave speed. Further, introducing $\mathbf{w}=\mathbf{R}^{-1} \mathbf{q}$ as the characteristic variable, the homogeneous form of Equation (1) can be diagonalized by the multiplication with $\mathbf{R}^{\mathbf{- 1}}$ to obtain the following form:

$$
\frac{\partial \mathbf{w}}{\partial t}+\Lambda \nabla \cdot \mathbf{w}=0
$$

The initial characteristic variable is defined as

$$
\stackrel{\circ}{\mathbf{w}}(x)= \begin{cases}\mathbf{R}^{-1} \mathbf{q}^{-}=\mathbf{w}^{-} & \text {for } x<0, \\ \mathbf{R}^{-1} \mathbf{q}^{+}=\boldsymbol{w}^{+} & \text {for } x>0 .\end{cases}
$$

Equation (8) shows that each characteristic variable $w_{i}(x, t)$ is decoupled and can be treated as an advection equation. The general solution of $w_{i}(x, t)$ is

$$
w_{i}(x, t)= \begin{cases}w_{i}^{-} & \text {for }\left(x-\lambda_{i} t\right)<0, \\ w_{i}^{+} & \text {for }\left(x-\lambda_{i} t\right)>0,\end{cases}
$$

where $\lambda_{i}$ is the wave velocity in the eigen-values matrix. This solution shows that the discontinuity emanating from $x=0$ is propagating along the characteristic curves $x=$ $\lambda_{i} t$. Now, if we define $p$ as the maximum value of $i$ for which $x-\lambda_{i} t>0$ at $(x, t)$, and transform it back to the original unknown variables by multiplying it with $\mathbf{R}$, the Riemann solution at the interface of the elements may be written as

$$
\mathbf{q}(x, t)=\sum_{i=1}^{p} w_{i}^{+} \mathbf{r}_{i}+\sum_{i=p+1}^{6} w_{i}^{-} \mathbf{r}_{i} .
$$

Equation (9) shows that the Riemann solution is a combination of eigen-vectors $\mathbf{r}_{i}$ with $w_{i}$ as the magnitude. To get better insight, the Riemann solution is illustrated in Figure 1, where six characteristic curves emanating from 
$x=0$ are drawn. The characteristics curves are related to the eigen-values $-c_{p},-c_{s}, c_{s}, c_{p}$. Notice that there are two coincident characteristic curves at $-c_{s}$ and $c_{s}$. The zero eigen-values are omitted since there is no contribution related to these eigen-vectors.

Based on Figure 1, we could divide the Riemann solution into six regions: $\mathbf{q}^{-}, \mathbf{q}^{\mathrm{I}}, \mathbf{q}^{\mathrm{II}}, \mathbf{q}^{\mathrm{III}}, \mathbf{q}^{\mathrm{IV}}, \mathbf{q}^{+}$. Each region represents the region of $(x, t)$ with the same solution. $\mathbf{q}^{-}$ is the solution of the first region where $\left(x+c_{p} t<0\right)$; $\mathbf{q}^{\mathrm{I}}$ is the solution of the second region where $\left(x+c_{p} t>0\right)$ and $\left(x+c_{s} t<0\right) ; \mathbf{q}^{\mathrm{II}}$ is the solution of the third region where $\left(x+c_{s} t\right)>0$ and $\left(x-c_{s} t<0\right)$; and so on. The $\mathbf{q}^{\mathrm{I}}, \mathbf{q}^{\mathrm{II}}, \mathbf{q}^{\mathrm{III}}, \mathbf{q}^{\mathrm{IV}}$ are known as the intermediate states [12]. In these regions, the solution is unknown and should be determined from the known initial values $\mathbf{q}^{+}$and $\mathbf{q}^{-}$. From Equation (9), it is known that across each $i$-th characteristic curve, the jump of the solution $\mathbf{q}$ can be defined as $\left(w_{i}^{+}-w_{i}^{-}\right) \mathbf{r}_{i}$. Hence, the total jump between $\mathbf{q}^{+}$and $\mathbf{q}^{-}$is

$$
\begin{aligned}
\mathbf{q}^{+}-\mathbf{q}^{-}= & \left(w_{1}^{+}-w_{1}^{-}\right) \mathbf{r}_{1}+\left(w_{2}^{+}-w_{2}^{-}\right) \mathbf{r}_{2} \\
& +\left(w_{3}^{+}-w_{3}^{-}\right) \mathbf{r}_{3}+\left(w_{4}^{+}-w_{4}^{-}\right) \mathbf{r}_{4} \\
& +\left(w_{5}^{+}-w_{5}^{-}\right) \mathbf{r}_{5}+\left(w_{6}^{+}-w_{6}^{-}\right) \mathbf{r}_{6}
\end{aligned}
$$

This jump between regions is called the Rankine-Hugoniot jump condition. This condition is essential to derive the solution for the intermediate states. Further, we define $\alpha_{i}=\left(w_{i}^{+}-w_{i}^{-}\right)$to have a more compact notation. Thus, Equation (10) can be written as

$$
\begin{aligned}
\mathbf{q}^{+}-\mathbf{q}^{-}= & \alpha_{1} \mathbf{r}_{1}+\alpha_{2} \mathbf{r}_{2}+\alpha_{3} \mathbf{r}_{3} \\
& +\alpha_{4} \mathbf{r}_{4}+\alpha_{5} \mathbf{r}_{5}+\alpha_{6} \mathbf{r}_{6}
\end{aligned}
$$

Moreover, $\alpha$ can be defined from the jump condition as

$$
\alpha=\mathbf{R}^{-1}\left(\mathbf{q}^{+}-\mathbf{q}^{-}\right) .
$$

Having $\alpha$ at hand, now we can obtain the intermediate states at any position and time. In the DG method, we assume the location of discontinuity $(x=0)$ as the location of the interface between elements. To calculate the numerical fluxes, we need to obtain the intermediate states located at $(x=0, t>0)$, which in this case are the $\mathbf{q}^{\mathrm{II}}$ or the $\mathbf{q}^{\mathrm{III}}$ regions, both expressions are equal. Based on Equations (9) and (11), these regions can be written as

$$
\mathbf{q}^{\mathrm{II}}=\mathbf{q}^{-}+\alpha_{1} \mathbf{r}_{1}+\alpha_{2} \mathbf{r}_{2}+\alpha_{3} \mathbf{r}_{3},
$$

or

$$
\mathbf{q}^{\mathrm{III}}=\mathbf{q}^{+}-\alpha_{6} \mathbf{r}_{6}-\alpha_{5} \mathbf{r}_{5}+\alpha_{4} \mathbf{r}_{4}
$$

We take $\mathbf{q}^{\mathrm{II}}$ as the region of interest, and the upwind numerical fluxes are expressed as $\mathbf{f}^{*}=\mathbf{A} \mathbf{q}^{\mathrm{II}}$. The details of $\mathbf{f}^{*}$ are given in the Appendix.

\subsection{Point force excitation}

In order to insert force into a domain, an external source can be introduced by imposing time-domain stress in some area of the domain boundary [14] or by having the body

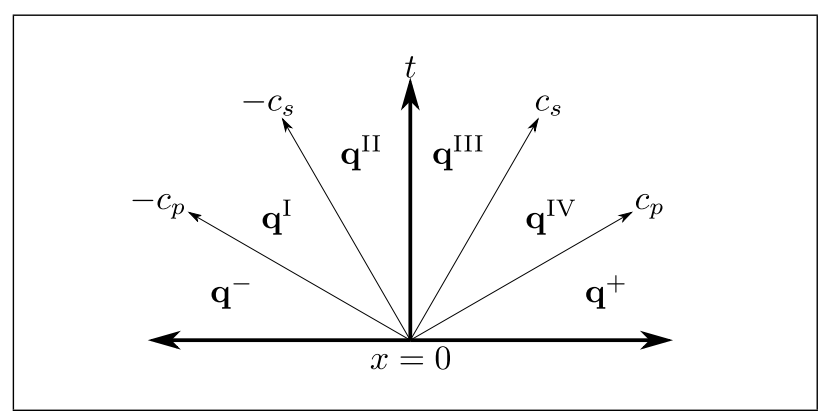

Figure 1. Riemann solution illustration.

force vector $\mathbf{g}$ [ $\mathrm{N} / \mathrm{kg}$ ] at the right-hand side of the LE equations.

In this work, we apply the latter approach. The point body force is approximated using the Lagrange interpolation polynomials, and the point body force is solely excited at $z$-direction $g_{z}(\boldsymbol{x}, t)$ as

$$
g_{z}(\boldsymbol{x}, t)=g_{z}(t) \cdot \delta\left(\boldsymbol{x}-\boldsymbol{x}_{0}\right)
$$

where $g_{z}(t)$ is a time domain function, $\delta(\boldsymbol{x})$ is the spatial Dirac delta function, and $x_{0}$ is the excitation location. The point body force can be approximated by exciting one nodal basis in an element. However, it could create forces with certain directivity. To overcome this problem, we distribute the same $g_{z}(t)$ on several elements that have a vertex located at $\boldsymbol{x}_{0}$. After applying the same body force in each element, the total force $F(t)[\mathrm{N}]$ in the computational domain can be computed as

$$
\boldsymbol{F}(t)=\rho \sum_{k=1}^{K_{p}} F^{k}(t)=\rho \sum_{k=1}^{K_{p}} \int_{D_{k}} g_{z}^{k}(\boldsymbol{x}, t) \mathrm{d} \boldsymbol{x}
$$

where $K_{p}$ is the number of elements around the excitation location, and $g_{z}^{k}(\boldsymbol{x}, t)$ is the body force function in each element $k$.

The integration in Equation (13) can be conducted in each element by transforming it into a standard tetrahedral element (I) as

$$
F^{k}(t)=\rho g_{z}(t) \boldsymbol{J}^{k} \int_{\mathbf{I}} \sum_{i=1}^{N_{p}} g_{z}^{k}\left(\boldsymbol{\xi}_{i}\right) \varphi_{i}(\boldsymbol{\xi}) \mathrm{d} \boldsymbol{\xi}
$$

where $J^{k}$ is the transformation Jacobian of element $D_{k}, \xi_{i}$ are the optimised LGL points at $\mathbf{I}$, and $\boldsymbol{\xi}$ is the reference coordinate $\left(\xi_{1}, \xi_{2}, \xi_{3}\right)$. I is defined as

$$
\mathbf{I}=\left\{\left(\xi_{1}, \xi_{2}, \xi_{3}\right) \mid \xi_{1}, \xi_{2}, \xi_{3} \geqslant-1 ; \xi_{1}+\xi+\xi_{3} \leqslant 1\right\} .
$$

Since we excite only one nodal basis in each element, the sum over index $i$ in Equation (14) only has a value at one nodal point. However, this nodal point might be different for each element, so we keep the index $i$.

To perform the integration of Equation (14), we need to have the closed-form of $\varphi_{i}(\xi)$, and it can be expressed by the 3-D orthogonal basis function $\Psi_{j}(\xi)$ such as defined 
in Equation (3). By this relation, Equation (14) can be rewritten as

$$
F^{k}(t)=\rho g_{z}(t) J^{k} \sum_{i=1}^{N_{p}} \sum_{j=1}^{N_{p}} g_{z}^{k}\left(\xi_{i}\right)\left(\mathrm{V}^{T}\right)_{i j}^{-1} \int_{\mathbf{I}} \Psi_{j}(\xi) \mathrm{d} \xi
$$

The modal basis $\Psi_{j}(\xi)$ is defined following Hesthaven [10] as

$$
\begin{aligned}
& \Psi_{j}\left(\xi_{1}, \xi_{2}, \xi_{3}\right)=\sqrt{8} P_{a}^{(0,0)}\left(\eta_{1}\right) P_{b}^{(2 a+1,0)}\left(\eta_{2}\right) \\
& \cdot P_{c}^{(2 a+2 b+2,0)}\left(\eta_{3}\right)\left(1-\eta_{2}\right)^{a}\left(1-\eta_{3}\right)^{a+b}, \\
& \eta_{1}=\frac{-2\left(1+\xi_{1}\right)}{\xi_{2}+\xi_{3}}, \quad \eta_{2}=\frac{2\left(1+\xi_{1}\right)}{1-\xi_{3}}-1, \quad \eta_{3}=\xi_{3},
\end{aligned}
$$

where $P_{a}^{(\alpha, \beta)}$ are the Jacobi polynomials with weight $\alpha$ and $\beta$, and $a+b+c \leq N$. The $\left(\eta_{1}, \eta_{2}, \eta_{3}\right)$ are the collapsed coordinates and they vary between $[-1,1]$. By expressing the $\Psi_{j}(\xi)$ using the collapsed coordinates, the integration in Equation (15) is separable and can be presented as

$$
\begin{aligned}
\int_{\mathbf{I}} \Psi_{j}(\xi) \mathrm{d} \xi= & \frac{1}{\sqrt{8}} \int_{-1}^{1} P_{a}^{(0,0)}\left(\eta_{1}\right) \mathrm{d} \eta_{1} \\
& \cdot \int_{-1}^{1}\left(1-\eta_{2}\right)^{a+1} P_{b}^{(2 a+1,0)}\left(\eta_{2}\right) \mathrm{d} \eta_{2} \\
& \cdot \int_{-1}^{1}\left(1-\eta_{3}\right)^{a+b+2} P_{c}^{(2 a+2 b+2,0)}\left(\eta_{2}\right) \mathrm{d} \eta_{3} .
\end{aligned}
$$

It should be noted that the transformation Jacobian between reference coordinate and the collapsed coordinate is included in Equation (16). Finally, the Equation (16) can be integrated using the Gauss-Jacobi quadrature to have the total force that is inserted into the computational domain. To summarise, the time-domain body force is given at a specific vertex in several elements around the point force excitation; afterwards, the Gauss-Jacobi quadrature formula is applied to compute the total force. The total force is used to calculate the plate mobility of the nodal DG solution such as configured in the next sections.

\section{3-D plate problem with forced vibration}

\subsection{Rectangular plate configuration}

We consider a rectangular plate represented by a cuboid computational domain of sizes $[0,1.8] \times[0,1.2] \times[0, h] \mathrm{m}$, with $h$ is the plate thickness. The plate is made of concrete with Young's modulus $(E) 33.7 \mathrm{GPa}$, density $(\rho)$ $2300 \mathrm{~kg} / \mathrm{m}^{3}$, and Poisson's ratio (v) 0.3 . The corresponding Lamé parameters are $\lambda=E v /(1+v)(1-2 v)$ and $\mu=E / 2(1+v)$.

The first objective of the rectangular plate configuration is to compute the transfer mobilities of the rectangular plates with the mechanical properties as mentioned above with different BCs and thicknesses. The transfer mobility is computed between the force located at $\mathrm{F}=[0.3,0.9, h] \mathrm{m}$ and the velocity located at $\mathrm{R}=[1.6$, $0.3, h] \mathrm{m}$. The plate thickness are vary of $h=[0.12,0.08$,

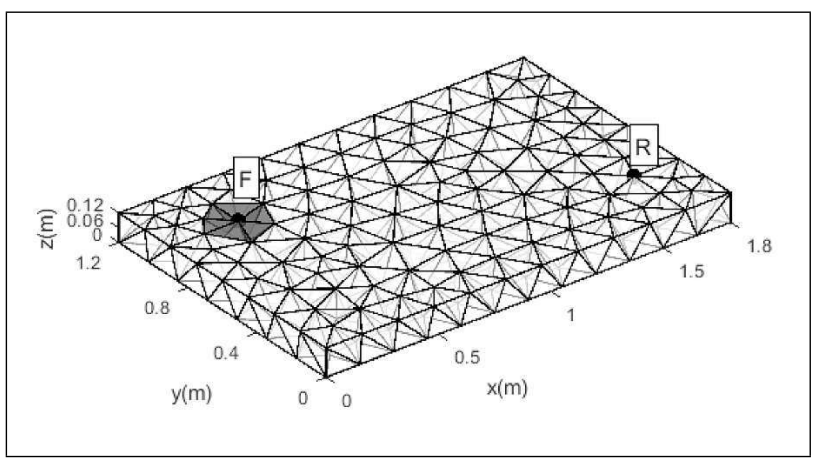

Figure 2. Rectangular plate computational domain with the force and receiver locations for $h=0.12 \mathrm{~m}$.

Table I. Numerical settings of the rectangular plate configuration.

\begin{tabular}{|ccccc|}
\hline$h$ & $K$ & DoF & $C_{C F L}$ & $\Delta t$ \\
\hline $0.12 \mathrm{~m}$ & 641 & 6410 & 1 & $2.86 \cdot 10^{-6}$ \\
$0.08 \mathrm{~m}$ & 630 & 6300 & 1 & $2.02 \cdot 10^{-6}$ \\
$0.04 \mathrm{~m}$ & 616 & 6160 & 0.7 & $7.09 \cdot 10^{-7}$ \\
\hline
\end{tabular}

0.04] $\mathrm{m}$, and each thickness has three sets of BCs: S-S-SS, S-S-S-C, and S-S-S-F BCs. Each set of the plate BCs is described in Section 4.1.1.

The second objective of the rectangular plate configuration is to analyse the accuracy of transfer mobility around the point force excitation location. The plate with S-S$\mathrm{S}-\mathrm{F} \mathrm{BCs}$ and $h=0.12 \mathrm{~m}$ is taken as an example to investigate the accuracy. The transfer mobilities are taken between the force located at $\mathrm{F}$ and several velocities located at R1 $=[0.3,0.85, h] \mathrm{m}, \mathrm{R} 2=[0.25,0.85, h] \mathrm{m}$, $\mathrm{R} 3=[0.35,0.95, h] \mathrm{m}$, and $\mathrm{R} 4=[0.4,0.9, h] \mathrm{m}$.

For both objectives, the body force $g_{z}(t)$ is given in the form of Ricker wavelet as follows

$$
g_{z}(t)=\left(0.5-\left(\pi f_{c}\left(t-t_{d}\right)\right)^{2}\right) e^{\left(\pi f_{c}\left(t-t_{d}\right)\right)^{2}},
$$

with the center frequency $f_{c}=500 \mathrm{~Hz}$ and the center time $t_{d}=5 \mathrm{~ms}$. This center frequency is taken to have the force excitation up to $1 \mathrm{kHz}$.

To solve the configuration, we discretise the plate using unstructured tetrahedral elements generated by the Gambit mesh generator. The computational domain of the plate with $h=0.12 \mathrm{~m}$ is shown in Figure 2. We mark the force (F) and velocity (R) locations, and highlight the elements that are associated with the point force with red colour. Afterwards, we ascribe the BCs to each computational faces such as presented in Section 4.1.1. The nodal basis of $N=3$ is used for all cases of the rectangular plate configuration. Finally, the details of the mesh and time steps are given in the Table I.

\subsubsection{Rectangular plate boundary conditions}

To complete the problem definition, we ascribe the BC to each plate face. There are six sets of rectangular plate BCs that have exact mode shapes and eigenvalues based on plate theories $[19,15]$. In this work, we consider three 
sets of BCs which we identify as the S-S-S-S plate, the SS-S-C plate, and the S-S-S-F plate. The labels refer to the plate faces at $x=0, y=0, x=1.8 \mathrm{~m}$, and at $y=1.2 \mathrm{~m}$, respectively. The indexes $\mathrm{S}, \mathrm{C}$, and $\mathrm{F}$ indicates the simply supported BC, the clamped $\mathrm{BC}$, and the free $\mathrm{BC}$, respectively. For all sets, the top and bottom faces at $z=h$ and $z=0$ are treated with free BCs which implies

$$
\tau_{x z}=\tau_{y z}=\tau_{z z}=0 .
$$

In the nodal DG method, this translates to the following conditions:

$$
\begin{array}{ll}
\tau_{x z}^{+}=-\tau_{x z}^{-}, & \tau_{x x}^{+}=\tau_{x x}^{-}, v_{x}^{+}=v_{x}^{-}, \\
\tau_{y z}^{+}=-\tau_{y z}^{-}, & \tau_{y y}^{+}=\tau_{y y}^{-}, v_{y}^{+}=v_{y}^{-}, \\
\tau_{z z}^{+}=-\tau_{z z}^{-}, & \tau_{x y}^{+}=\tau_{x y}^{-}, v_{z}^{+}=v_{z}^{-} .
\end{array}
$$

These settings set the stress components at the top and the bottom faces as in Equation (17).

\section{S-S-S-S plate}

The details considering the S-S-S-S plate on the 3-D domain can be found in the work of Srinivas et.al. [16]. According to Srinivas, to satisfy the simply supported BC, the plate face at $x=0$ and $x=1.8 \mathrm{~m}$ should have the following conditions:

$$
\begin{aligned}
& v_{y}=0, \tau_{x x}=0, \tau_{z z}=0, \\
& v_{z}=0, \tau_{y y}=0, \tau_{y z}=0 .
\end{aligned}
$$

In the nodal DG method, this BC is implemented as

$$
\begin{gathered}
v_{y}^{+}=-v_{y}^{-}, \tau_{x x}^{+}=-\tau_{x x}^{-}, \tau_{z z}^{+}=-\tau_{z z}^{-}, \\
v_{z}^{+}=-v_{z}^{-}, \tau_{y y}^{+}=-\tau_{y y}^{-}, \tau_{y z}^{+}=-\tau_{y z}^{-}, \\
v_{x}^{+}=v_{x}^{-}, \tau_{x y}^{+}=\tau_{x y}^{-}, \quad \tau_{x z}^{+}=\tau_{x z}^{-} .
\end{gathered}
$$

For the plate face at $y=0$ and $y=1.2 \mathrm{~m}$, it should have the following conditions:

$$
\begin{aligned}
& v_{x}=0, \tau_{x x}=0, \tau_{z z}=0, \\
& v_{z}=0, \tau_{y y}=0, \tau_{x z}=0 .
\end{aligned}
$$

In the nodal DG method, this BC is implemented as

$$
\begin{aligned}
& v_{x}^{+}=-v_{x}^{-}, \tau_{x x}^{+}=-\tau_{x x}^{-}, \tau_{z z}^{+}=-\tau_{z z}^{-}, \\
& v_{z}^{+}=-v_{z}^{-}, \tau_{y y}^{+}=-\tau_{y y}^{-}, \tau_{x z}^{+}=-\tau_{x z}^{-}, \\
& v_{y}^{+}=v_{y}^{-}, \tau_{x y}^{+}=\tau_{x y}^{-}, \tau_{y z}^{+}=\tau_{y z}^{-} .
\end{aligned}
$$

\section{S-S-S-C plate}

To obtain the S-S-S-C plate, the plate faces at $x=0$ and $x=1.8 \mathrm{~m}$ should satisfy Equation (19) and follow the nodal DG settings at Equation (20). Afterwards, the face at $y=0$ should satisfy Equation (21) and follow the implementation at Equation (22). For the face at $y=1.2 \mathrm{~m}$, the plate should satisfy the following conditions:

$$
v_{x}=v_{y}=v_{z}=0 .
$$

In the nodal DG method, this BC is implemented as

$$
\begin{aligned}
& \tau_{x x}^{+}=\tau_{x x}^{-}, \tau_{x z}^{+}=\tau_{x z}^{-}, v_{x}^{+}=-v_{x}^{-}, \\
& \tau_{y y}^{+}=\tau_{y y}^{-}, \tau_{y z}^{+}=\tau_{y z}^{-}, v_{y}^{+}=-v_{y}^{-}, \\
& \tau_{z z}^{+}=\tau_{z z}^{-}, \tau_{x y}^{+}=\tau_{x y}^{-}, v_{z}^{+}=-v_{z}^{-} .
\end{aligned}
$$

\section{S-S-S-F plate}

The S-S-S-F plate has the same settings as the S-S-S-C plate at the faces of $x=0, x=1.8 \mathrm{~m}$, and $y=0$. For the face at $y=1.2 \mathrm{~m}$, the plate should satisfy the following condition:

$$
\tau_{x y}=\tau_{y y}=\tau_{y z}=0 .
$$

In the nodal DG method, this BC is implemented as

$$
\begin{aligned}
& \tau_{x y}^{+}=-\tau_{x y}^{-}, \tau_{x x}^{+}=\tau_{x x}^{-}, v_{x}^{+}=v_{x}^{-}, \\
& \tau_{y y}^{+}=-\tau_{y y}^{-}, \quad \tau_{z z}^{+}=\tau_{z z}^{-}, \quad v_{y}^{+}=v_{y}^{-}, \\
& \tau_{y z}^{+}=-\tau_{y z}^{-}, \tau_{x z}^{+}=\tau_{x z}^{-}, v_{z}^{+}=v_{z}^{-} \text {. }
\end{aligned}
$$

\subsection{Rectangular plate analytical mobility}

To validate the numerical results from the nodal DG method, we compare the plate mobility with an exact mobility obtained from plate theories: classical plate theory (CPT) and first-order shear deformation plate theory (FSDT). The CPT is selected since it has been used extensively in building acoustics applications, and the FSDT is selected since it is more accurate than the CPT. We obtain the mode shapes and the natural frequencies from each plate theories and calculate the mobility by applying the modal summation method. The details of the CPT and the FSDT can be found in literature [17].

The mobility $(\mathrm{Y})$ between the force located at $\left(x_{0}, y_{0}\right)$ and the velocity $\left(v_{z}\right)$ located at $\left(x_{r}, y_{r}\right)$ can be written as

$$
\mathrm{Y}(\omega)=\mathrm{i} \omega \sum_{m=1}^{\infty} \sum_{n=1}^{\infty} \frac{F_{m n}\left(x_{0}, y_{0}\right) \Phi_{m n}\left(x_{r}, y_{r}\right)}{M\left(\omega_{m n}^{2}(1+j \zeta)-\omega^{2}\right)},
$$

where $F_{m n}$ is the modal force, $\Phi_{m n}$ is the mode shape, $M$ is the inertia component, $\omega_{m n}$ is the angular frequency of the natural mode $m n, \zeta$ is the structural damping, and $\omega$ is the angular frequency. The indexes $m$ and $n$ represent the mode shape in $x$-direction and $y$-direction, respectively. $F_{m n}$ is the projection of the force to the $m n$ natural mode shape. For the point force excitation, modal force can be written as

$$
F_{m n}=\frac{\Phi_{m n}\left(x_{0}, y_{0}\right)}{\int_{0}^{a} \int_{0}^{b} \Phi_{m n}^{2}(x, y) \mathrm{d} x \mathrm{~d} y},
$$

where $a$ and $b$ are the plate length and width. According to the CPT, the S-S-S-S plate mode shape and natural angular frequency can be written as

$$
\begin{aligned}
\Phi_{m n}(x, y) & =\sin \left(\alpha_{m} x\right) \sin \left(\beta_{n} y\right), \\
\omega_{m n}^{2} & =D\left(\alpha_{m}^{2}+\beta_{n}^{2}\right)^{2} / M,
\end{aligned}
$$

where $\alpha_{m}=m \pi / a, \beta_{n}=n \pi / b$, the bending stiffness $D=$ $\left(E h^{3}\right) /\left(12\left(1-v^{2}\right)\right)$, and the inertia component $M=\rho h$. In practical applications, often plate rotary inertia is often omitted in CPT. However, it could over-estimate the plate natural frequencies. For the S-S-S-S plate, we could include the effect of rotary inertia to calculate the mobility by defining the inertia component in Equation (27) as

$$
M=\rho h+\frac{\rho h^{3}}{12}\left(\alpha_{m}^{2}+\beta_{n}^{2}\right) .
$$


For the mobility according to FSDT, Wang has presented an exact relationship between the natural angular frequency obtained by the CPT and the FSDT [15]. This relation has the mode shape as in Equation (27) and the natural angular frequency $\omega_{m n}^{F S D T}$ is as follows:

$$
\begin{aligned}
\left(\omega_{m n}^{F S D T}\right)^{2} & =\frac{6 K_{s} G}{\rho h^{2}}\left(C_{m n}-\sqrt{\left.C_{m n}^{2}-\frac{\rho h^{2}\left(\omega_{m n}^{2}\right)}{3 K_{s} G}\right)},\right. \\
C_{m n} & =1+\frac{\omega_{m n} h^{2}}{12} \sqrt{\frac{\rho h}{D}}\left(1+\frac{2}{K_{s}^{2}(1-v)}\right),
\end{aligned}
$$

where $K_{s}$ is the shear correction factor. By this relation, we obtain the FSDT mobility by replacing $\omega_{m n}^{2}$ with $\left(\omega_{m n}^{F S D T}\right)^{2}$ in Equation (25).

Concerning the S-S-S-C and S-S-S-F plates, we compute the mobility based on the CPT adopting the work of Gorman [18] and based on the FSDT adopting the work of Wang et al. [15]. These authors provide the exact natural frequency and the mode shape of the plates. The interested reader is referred to the work of Gorman[18], Hashemi [19], or Wang [15] for further information.

\subsection{L-shaped plate configuration}

For more complex plate vibration problems, we compute the mobility of a 3-D L-shaped plate. The problem domain is a combination of two cuboids of $h=$ $0.12 \mathrm{~m}$. The first cuboid is of size $[-h / 2,1.8] \times$ $[0,1.2] \times[-h / 2, h / 2] \mathrm{m}$, and the second cuboid is of size $[-h / 2, h / 2] \times[0,1.2] \times[-h / 2,1.8] \mathrm{m}$. The cuboids have an overlap at $[-h / 2, h / 2] \times[0,1.2] \times[-h / 2, h / 2] \mathrm{m}$.

The objective is to compute the transfer mobilities between the point force located at $\mathrm{F}=[1.52,0.21, h / 2]$, and the velocity $\left(v_{z}\right)$ at $\mathrm{R} 1=[0.4,0.2, h / 2] \mathrm{m}, \mathrm{R} 2=$ $[h / 2,0.6,0.4] \mathrm{m}$, and R3 $=[h / 2,1,0.8] \mathrm{m}$. Figure 3 shows the computational domain, the point force location, and the velocity locations. To complete the formulation, the BCs are defined at each plate face. The faces at $x=1.8 \mathrm{~m}$, $x=-h / 2 \mathrm{~m}$, and $x=h / 2 \mathrm{~m}$ are the free BCs, this implies

$$
\tau_{x x}=\tau_{x y}=\tau_{x z}=0 .
$$

In the nodal DG method, this BC is implemented as

$$
\begin{aligned}
& \tau_{x x}^{+}=-\tau_{x x}^{-}, \tau_{y y}^{+}=\tau_{y y}^{-}, \quad v_{x}^{+}=v_{x}^{-}, \\
& \tau_{x y}^{+}=-\tau_{x y}^{-}, \tau_{z z}^{+}=\tau_{z z}^{-}, \quad v_{y}^{+}=v_{y}^{-}, \\
& \tau_{x z}^{+}=-\tau_{x z}^{-}, \tau_{y z}^{+}=\tau_{y z}^{-}, \quad v_{z}^{+}=v_{z}^{-} .
\end{aligned}
$$

The faces at $z=-h / 2 \mathrm{~m}, z=h / 2 \mathrm{~m}$, and $z=1.8 \mathrm{~m}$ are also the free BCs. The conditions are following Equation (17), and are implemented in the nodal DG following the Equation (18). Finally, the faces at $y=0$ and $y=1.2$ have simply supported BCs. The conditions are following Equation (21), and are implemented in the nodal DG method following the Equation (22). In this work, the Lshaped plate is discretized with $K=3289$ which equals to $\mathrm{DoF}=65780$.

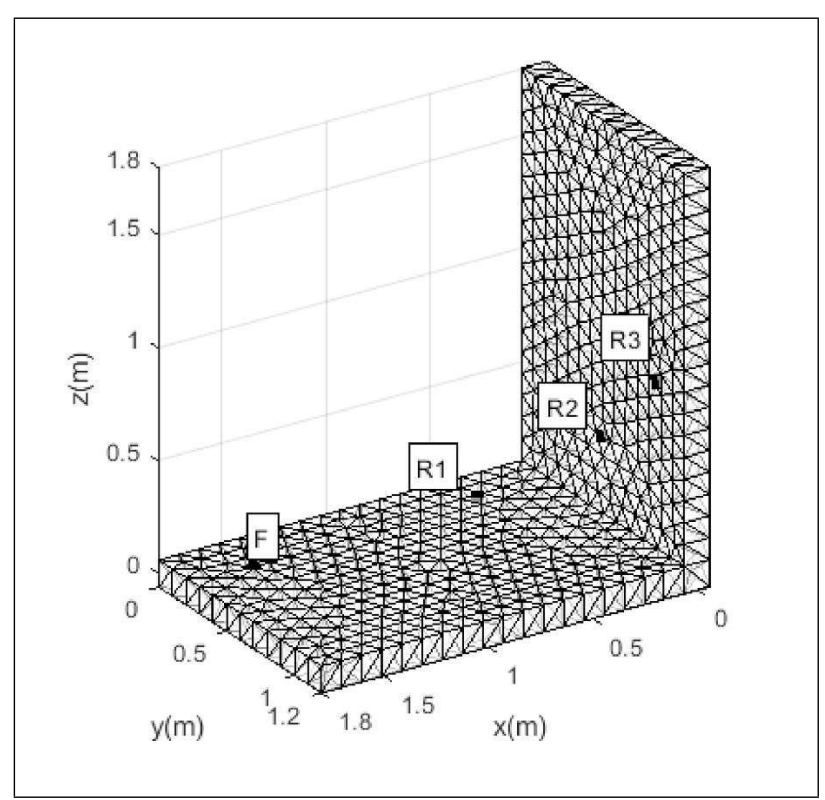

Figure 3. L-shaped plate domain with force and receiver locations for $h=0.12 \mathrm{~m}$.

\section{Results and discussions}

In this section, we show the plate mobilities for the various configurations. We obtain the spectra of the force and the velocity from the nodal DG solution to compute the plates mobilities. The spectra are obtained from a one-second time-domain signal, windowed in the last 0.5 seconds using a Hann window to reduce the Gibbs effect. Moreover, each spectrum is processed to have a $1 \mathrm{~Hz}$ resolution. Afterwards, we compare the nodal DG mobility with the CPT and the FSDT mobilities. It should be noted that a very weak constant damping $(0.1 \%)$ is given into the CPT and the FSDT to bound the amplitudes of the mobility. Moreover, we use a value of 0.86667 as the shear correction factor for the FSDT model following Hashemi [19]. Sections 5.1-5.3 show the rectangular plate mobilities for various thickness and BCs, section 5.4 shows the mobilities of the S-S-S-F plate for velocities near the excitation point, and section 5.5 shows the L-shaped plate mobilities.

\subsection{S-S-S-S plate}

Figures 4-6 show the mobilities of the S-S-S-S plate with different thicknesses. The solid thick lines are the mobilities obtained by the nodal DG method; the thick dash-dot lines are the mobilities obtained by the FSDT; the solid thin lines are the mobilities obtained by the CPT, and the thin dashed lines are the mobilities obtained by CPT with the rotary inertia included (CPT w/ RI). The rotary inertia inclusion is shown only for the S-S-S-S plate to show its significance to the results.

From Figures 4-6, we can observe that the plate bending stiffness is lowered as the thickness is reduced. It is shown by the reduction of the first natural frequency. At the resonance frequencies, we can see that the nodal DG mobility has a higher quality factor ( $\mathrm{Q}$ factor) compared to the other mobilities. The high $\mathrm{Q}$ factor shows that the energy is conserved well throughout the simulation. Overall, the com- 


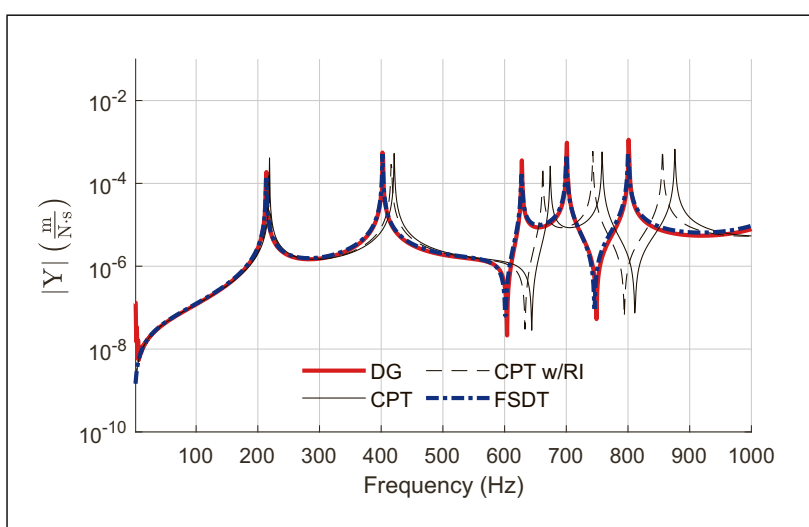

Figure 4. S-S-S-S Plate mobility for $h=0.12 \mathrm{~m}$.

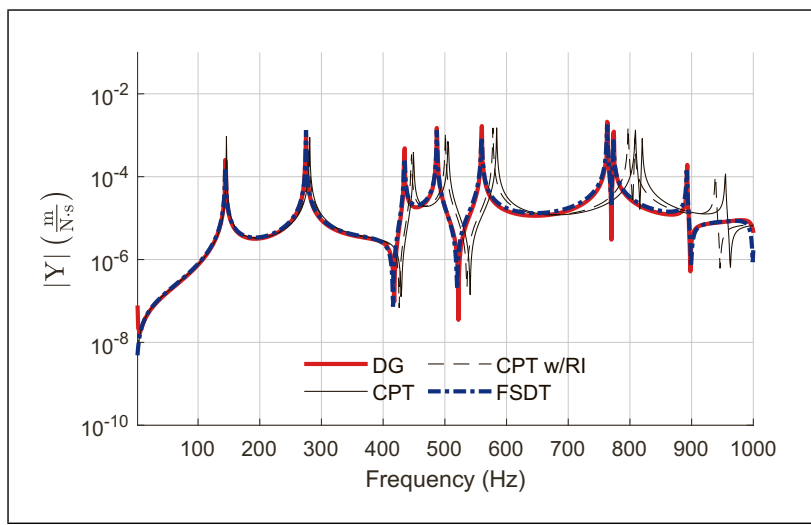

Figure 5. S-S-S-S Plate mobility for $h=0.08 \mathrm{~m}$.

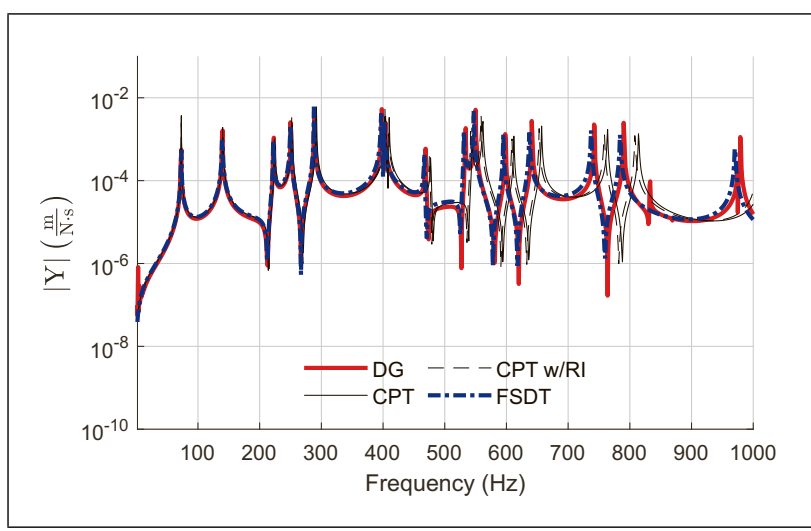

Figure 6. S-S-S-S Plate mobility for $h=0.04 \mathrm{~m}$.

parison shows that the nodal DG mobility has an excellent agreement with FSDT mobility. We tabulate the resonance frequencies of the plate in Tables II-IV. Compared to the FSDT, the resonance frequency differences are between 0 to $1 \mathrm{~Hz}$ for every thickness. On the other hand, the nodal DG mobility shows significant resonance frequency differences in comparison with the CPT. The differences are varying between 5 to $75 \mathrm{~Hz}$ for the plate with $h=0.12 \mathrm{~m}$; 2 to $62 \mathrm{~Hz}$ for the plate with $h=0.08 \mathrm{~m}$; and 0 to $24 \mathrm{~Hz}$ for the plate with $h=0.04 \mathrm{~m}$. Furthermore, the differences are reduced if the effect of rotary inertia is included in the CPT model, where the differences are varying between 4
Table II. Resonance frequencies [Hz] for $h=0.12 \mathrm{~m}$ up to $1 \mathrm{kHz}$.

\begin{tabular}{|cccc|}
\hline DG & CPT & CPT w/ RI & FSDT \\
\hline 214 & 219 & 218 & 214 \\
402 & 421 & 416 & 402 \\
628 & 674 & 662 & 628 \\
701 & 758 & 743 & 700 \\
801 & 876 & 856 & 800 \\
\hline
\end{tabular}

Table III. Resonance frequencies [Hz]for $h=0.08 \mathrm{~m}$ up to $1 \mathrm{kHz}$.

\begin{tabular}{|cccc|}
\hline DG & CPT & CPT w/ RI & FSDT \\
\hline 144 & 146 & 146 & 144 \\
275 & 281 & 279 & 275 \\
435 & 449 & 446 & 435 \\
487 & 505 & 501 & 487 \\
560 & 584 & 578 & 560 \\
763 & 809 & 797 & 763 \\
774 & 820 & 808 & 773 \\
893 & 955 & 939 & 892 \\
\hline
\end{tabular}

Table IV. Resonance frequencies [Hz] for $h=0.04 \mathrm{~m}$ up to $1 \mathrm{kHz}$.

\begin{tabular}{|cccc|}
\hline DG & CPT & CPT w/ RI & FSDT \\
\hline 73 & 73 & 73 & 73 \\
140 & 140 & 140 & 140 \\
223 & 225 & 224 & 223 \\
250 & 253 & 252 & 250 \\
289 & 292 & 291 & 289 \\
398 & 404 & 403 & 398 \\
404 & 410 & 409 & 404 \\
469 & 477 & 475 & 469 \\
534 & 545 & 542 & 534 \\
550 & 562 & 559 & 550 \\
598 & 612 & 609 & 598 \\
641 & 657 & 653 & 641 \\
742 & 764 & 759 & 742 \\
790 & 814 & 808 & 790 \\
\hline
\end{tabular}

to $55 \mathrm{~Hz}$ for the plate with $h=0.12 \mathrm{~m} ; 2$ to $46 \mathrm{~Hz}$ for the plate with $h=0.08 \mathrm{~m}$; and 0 to $18 \mathrm{~Hz}$ for the plate with $h=0.04 \mathrm{~m}$. It is important to notice that the bending wavelength for the S-S-S-S plate with $h=0.12 \mathrm{~m}$ at $1 \mathrm{kHz}$ is $0.934 \mathrm{~m}$. It indicates that the thickness is well below $1 / 6$ of the wavelength for every thickness taken. This principle has been a rule of thumb to use CPT in building acoustics application; however, considerable errors especially for high frequency are present even for the thinner plate.

\subsection{S-S-S-C plate}

Figures 7-9 show the mobilities of the S-S-S-C plate with different thicknesses. We can see that the bending stiffness of the S-S-S-C plate is higher than the S-S-S-S plate. The increment is shown by the resonance frequencies shift to higher frequencies. This shift occurs as a result of one 


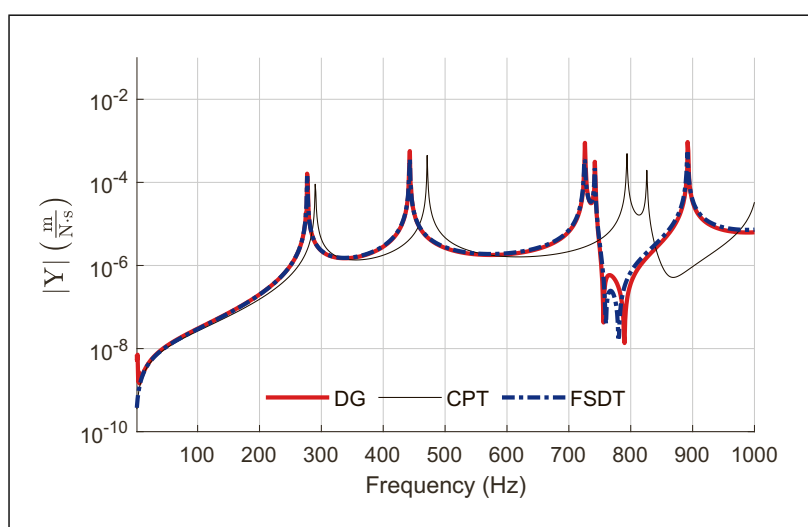

Figure 7. S-S-S-C Plate mobility for $h=0.12 \mathrm{~m}$.

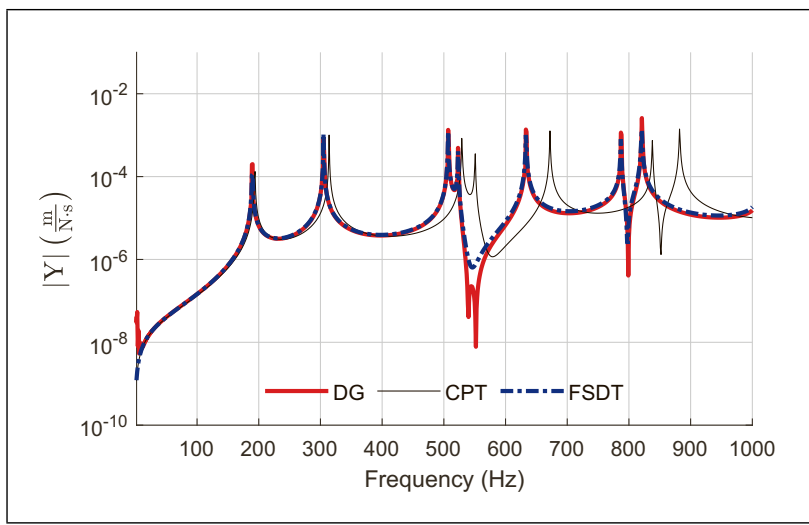

Figure 8. S-S-S-C Plate mobility for $h=0.08 \mathrm{~m}$.

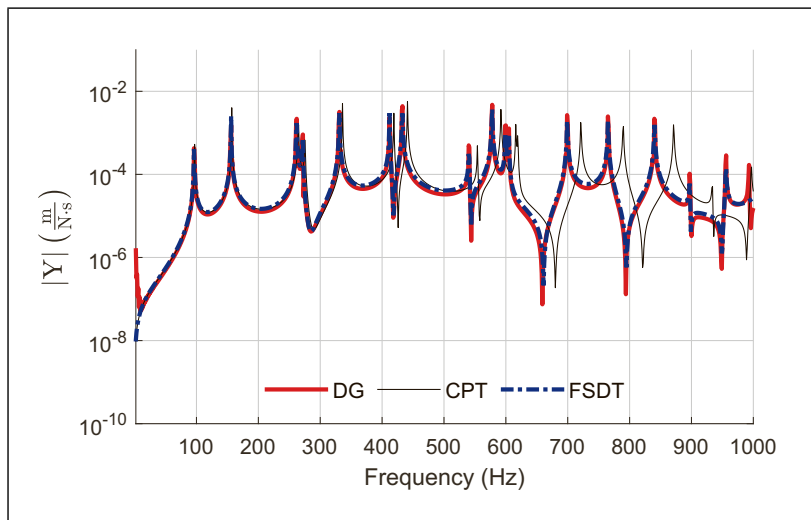

Figure 9. S-S-S-C Plate mobility for $h=0.04 \mathrm{~m}$.

plate face is clamped instead of simply supported. Tables $\mathrm{V}-\mathrm{VII}$ show the resonance frequencies of each plate thickness. Moreover, similar to the results obtained by the S-SS-S plate, the comparison shows that the nodal DG mobility has an excellent agreement with FSDT mobility. The resonance frequency differences are between 0 to $1 \mathrm{~Hz}$ for every thickness. On the other hand, the comparison with the CPT shows that differences are varying between 13 to $84 \mathrm{~Hz}$ for the plate with $h=0.12 \mathrm{~m} ; 5$ to $61 \mathrm{~Hz}$ for the plate with $h=0.08 \mathrm{~m}$; and 1 to $41 \mathrm{~Hz}$ for the plate with $h=0.04 \mathrm{~m}$.
Table V. Resonance frequencies [Hz] for $h=0.12 \mathrm{~m}$ up to $1 \mathrm{kHz}$.

\begin{tabular}{|ccc|}
\hline DG & CPT & FSDT \\
\hline 277 & 290 & 277 \\
443 & 471 & 443 \\
726 & 794 & 726 \\
742 & 826 & 742 \\
\hline
\end{tabular}

Table VI. Resonance frequency [Hz] for $h=0.08 \mathrm{~m}$ up to $1 \mathrm{kHz}$.

\begin{tabular}{|ccc|}
\hline DG & CPT & FSDT \\
\hline 189 & 194 & 190 \\
305 & 314 & 305 \\
507 & 529 & 507 \\
523 & 551 & 523 \\
633 & 672 & 633 \\
787 & 838 & 787 \\
821 & 882 & 821 \\
\hline
\end{tabular}

Table VII. Resonance frequency [Hz] for $h=0.04 \mathrm{~m}$ up to $1 \mathrm{kHz}$.

\begin{tabular}{|ccc|}
\hline DG & CPT & FSDT \\
\hline 96 & 97 & 96 \\
156 & 157 & 156 \\
262 & 265 & 262 \\
272 & 275 & 272 \\
331 & 336 & 331 \\
412 & 419 & 412 \\
433 & 441 & 433 \\
540 & 554 & 540 \\
578 & 592 & 578 \\
600 & 616 & 599 \\
605 & 619 & 605 \\
699 & 721 & 699 \\
765 & 790 & 765 \\
840 & 871 & 840 \\
897 & 934 & 897 \\
956 & 997 & 956 \\
\hline
\end{tabular}

\subsection{S-S-S-F plate}

Figures 10-12 show the mobilities of the S-S-S-F plate with different thicknesses. We can see that the bending stiffness of the S-S-S-F plate is lower than the S-S-S-S plate, and the resonance frequencies are shifted towards lower frequencies. This shift occurs because one plate face is free instead of suspending the transverse displacement. Tables VIII-X show the resonance frequencies of each plate thickness. Moreover, similar to the results obtained by the S-S-S-S plate, the comparison shows that the nodal DG mobility has an excellent agreement with the FSDT mobility. The resonance frequency differences are between 0 to $1 \mathrm{~Hz}$ for every thickness. On the other hand, the comparison with the CPT mobility shows that differences are varying between 2 to $88 \mathrm{~Hz}$ for the plate with $h=0.12 \mathrm{~m}$; 0 to $71 \mathrm{~Hz}$ for the plate with $h=0.08 \mathrm{~m}$; and 0 to $36 \mathrm{~Hz}$ for the plate with $h=0.04 \mathrm{~m}$. 


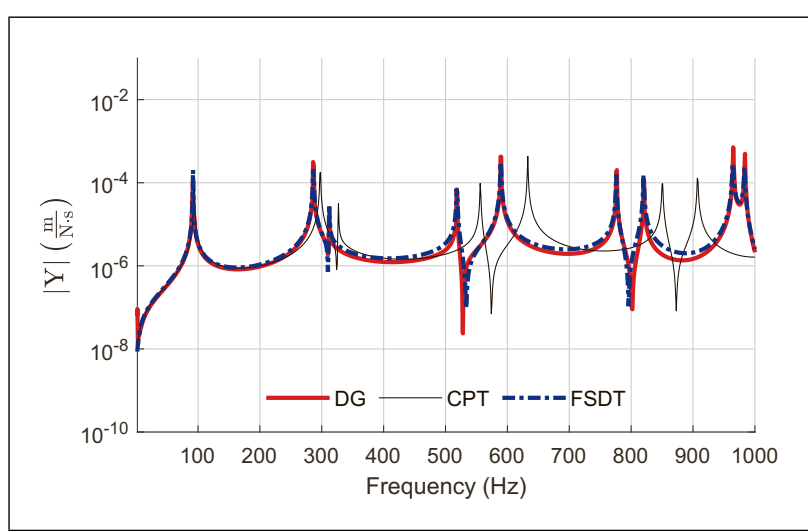

Figure 10. S-S-S-F Plate mobility for $h=0.12 \mathrm{~m}$.

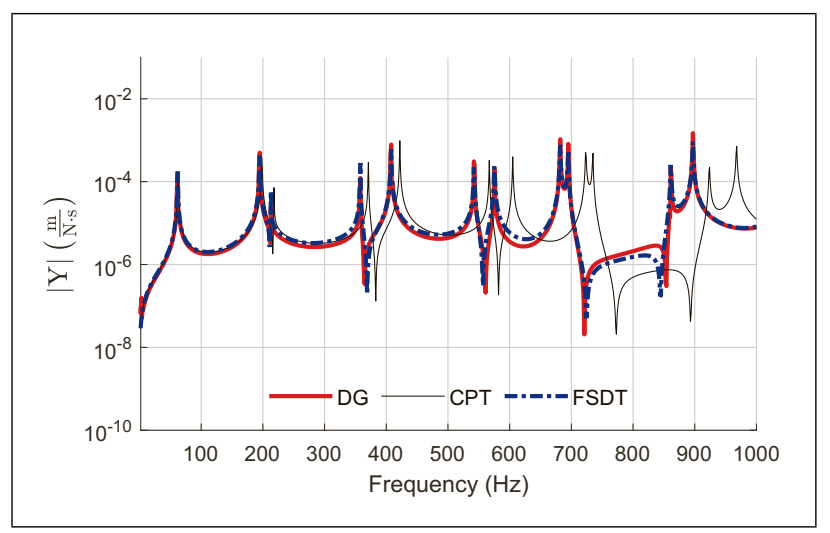

Figure 11. S-S-S-F Plate mobility for $h=0.08 \mathrm{~m}$.

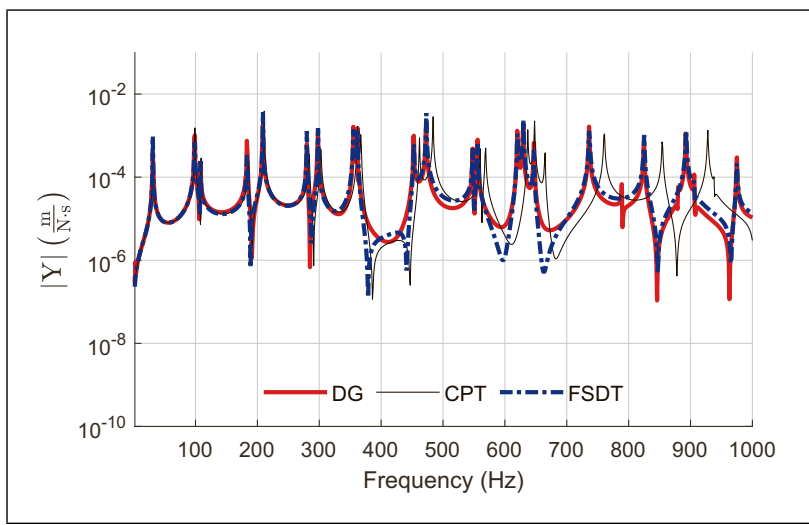

Figure 12. S-S-S-F Plate mobility for $h=0.04 \mathrm{~m}$.

\subsection{Mobility near to excitation point}

In this section, the mobilities of the S-S-S-F plate with $h=0.12 \mathrm{~m}$ around the excitation point are presented. It should be noticed that the R1-R4 positions are located at the highlighted elements near to the point $F$ such as shown in Figure 2, hence the velocity responses are taken at the same element where the force is excited. Figure 13 shows the mobilities at R1 and R2, and Figure 14 shows the mobilities at R3 and R4. Both figures show that the nodal DG mobilities are in an excellent agreement with the FSDT mobilities. The maximum difference of the resonance frequency is $1 \mathrm{~Hz}$ for all velocity positions. However, some
Table VIII. Resonance frequencies [Hz] for $h=0.12 \mathrm{~m}$ up to $1 \mathrm{kHz}$.

\begin{tabular}{|ccc|}
\hline DG & CPT & FSDT \\
\hline 92 & 94 & 92 \\
286 & 298 & 286 \\
312 & 327 & 312 \\
518 & 556 & 518 \\
589 & 633 & 589 \\
777 & 851 & 776 \\
820 & 908 & 820 \\
\hline
\end{tabular}

Table IX. Resonance frequency [Hz] for $h=0.08 \mathrm{~m}$ up to $1 \mathrm{kHz}$.

\begin{tabular}{|ccc|}
\hline DG & CPT & FSDT \\
\hline 62 & 62 & 62 \\
195 & 198 & 195 \\
213 & 218 & 213 \\
358 & 371 & 358 \\
408 & 422 & 408 \\
542 & 567 & 542 \\
575 & 605 & 575 \\
682 & 723 & 682 \\
695 & 735 & 695 \\
861 & 924 & 861 \\
897 & 968 & 897 \\
\hline
\end{tabular}

Table X. Resonance frequencies [Hz] for $h=0.04 \mathrm{~m}$ up to $1 \mathrm{kHz}$.

\begin{tabular}{|ccc|}
\hline DG & CPT & FSDT \\
\hline 31 & 31 & 31 \\
99 & 99 & 99 \\
108 & 109 & 108 \\
183 & 185 & 183 \\
209 & 211 & 209 \\
280 & 283 & 280 \\
298 & 302 & 298 \\
355 & 362 & 355 \\
362 & 367 & 362 \\
453 & 462 & 453 \\
473 & 484 & 473 \\
548 & 560 & 548 \\
556 & 569 & 556 \\
620 & 637 & 620 \\
630 & 648 & 630 \\
647 & 665 & 647 \\
736 & 761 & 736 \\
825 & 854 & 825 \\
892 & 928 & 893 \\
\hline
\end{tabular}

discrepancies happen at the anti-resonance frequencies. The anti-resonance frequencies have a lower Q factor compared to the FSDT anti-resonance frequencies. At R1, R2, $\mathrm{R} 3$, and R4, the anti-resonance frequency differences vary between $0-10 \mathrm{~Hz}, 1-7 \mathrm{~Hz}, 1-11 \mathrm{~Hz}$, and $1-11 \mathrm{~Hz}$, respectively. Moreover, we could observe that at the R4 location, the maximum discrepancy happens at $451 \mathrm{~Hz}$ instead of at the highest anti-resonance frequency of $977 \mathrm{~Hz}$. Al- 


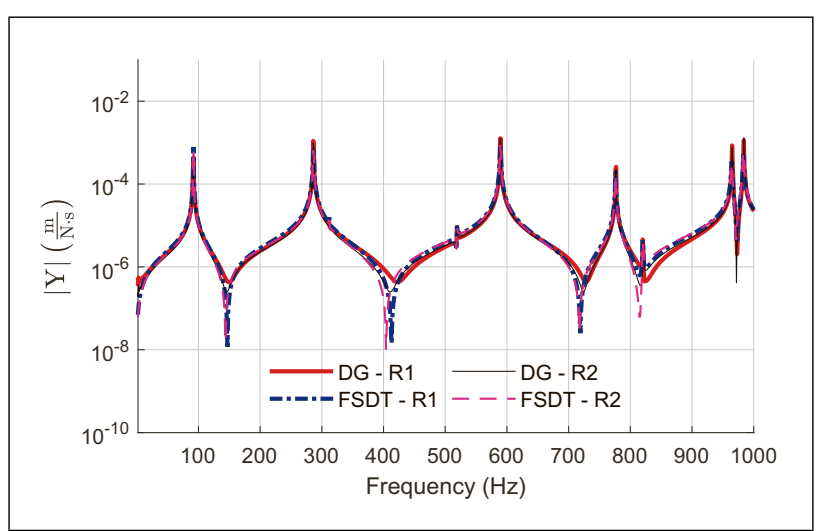

Figure 13. S-S-S-F Plate mobility at R1 and R2.

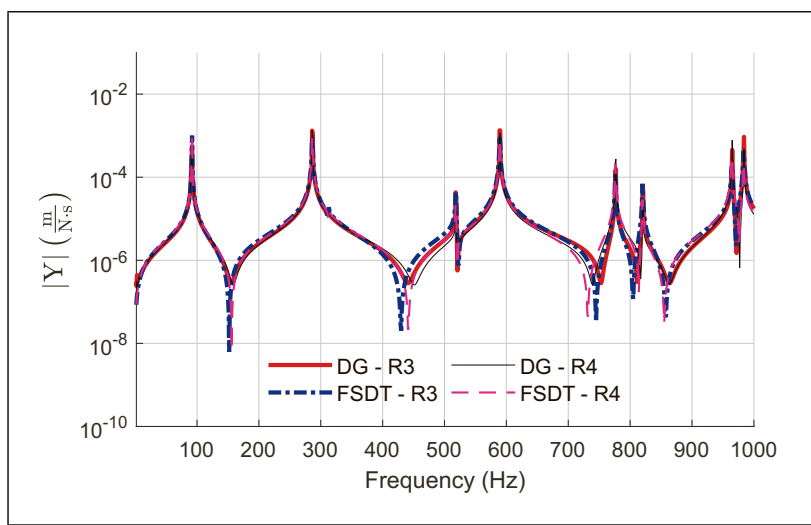

Figure 14. S-S-S-F Plate mobility at R3 and R4.

though the discrepancy at the anti-resonance frequency is not a significant issue for vibration problems, the agreement could be improved by refining the mesh near the excitation point.

\subsection{L-Plate}

In this section, we present the L-shaped plate mobilities between position F and R1 - R3 following the configuration of Section 4.3. The mobilities based on the CPT, the FSDT and the 3-D LE equation model are given to be compared with the mobility obtained by the nodal DG method. Kessissoglou presented the exact CPT mobility for this configuration, where the transverse and in-plane plate displacements are described in terms of a modal and travelling wave solution [20]. Moreover, the finite element method (FEM) in the frequency domain is used to obtain the mobilities based on the FSDT and the 3-D LE equations. The FEM model is implemented using COMSOL Multiphysics, where the FSDT is solved with a DoF number up-to $98 \cdot 10^{3}$, and the 3-D LE equations are solved with a DoF number up-to $264 \cdot 10^{3}$. With these numbers, the resonance frequencies have converged within a $1 \mathrm{~Hz}$ uncertainty. All mobilities are presented in Figures 15-17.

At all locations (R1-R3), Figures 15-17 show that the mobilities obtained by CPT, FSDT and nodal DG are in a good agreement at frequencies below $700 \mathrm{~Hz}$. We can observe that each resonance frequency of the nodal DG mobility has its counterpart in the CPT or the FSDT mobility.

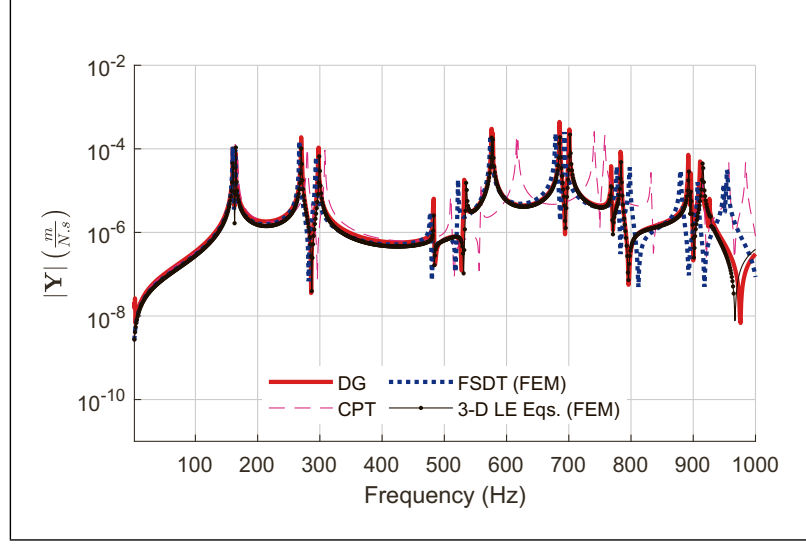

Figure 15. L-plate mobility at R1.

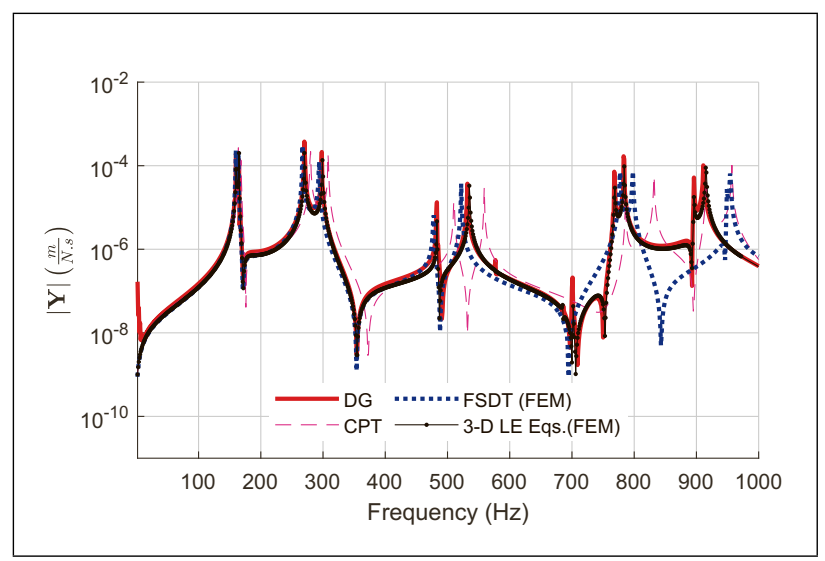

Figure 16. L-plate mobility at R2.

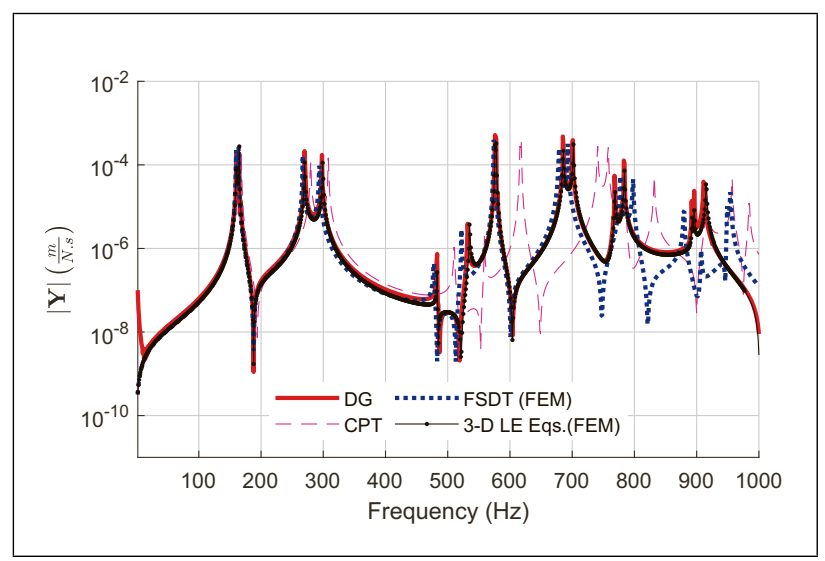

Figure 17. L-plate mobility at R3.

However, CPT mobility has higher resonance frequencies than the nodal DG mobility. These discrepancies are found as well in the rectangular plate case. The differences vary between $4 \mathrm{~Hz}$ and $58 \mathrm{~Hz}$ at R1, 3 and $73 \mathrm{~Hz}$ at R2, and 3 and $49 \mathrm{~Hz}$ at R3. Compared to the FSDT mobility, the nodal DG resonance frequencies have more difference in the L-shaped plate case than in the rectangular plate case. The resonance frequencies differences are up to $38 \mathrm{~Hz}$ at $\mathrm{R} 1$, up to $53 \mathrm{~Hz}$ at R2, and up to $42 \mathrm{~Hz}$ at R3. At R2 location, one also could notice that the anti-resonance trend is 
not similar between the nodal DG and the CPT/FSDT mobilities at around $700 \mathrm{~Hz}$. Above $700 \mathrm{~Hz}$, the similarities between the nodal DG mobility and the CPT/FSDT mobilities are lower due to the resonance or the anti-resonance location.

For further validation on the nodal DG solution, we show the mobilities of the 3-D LE equtions that are calculated by FEM in Figures 15-17. The mobilities from the nodal DG method and FEM show an excellent agreement with a maximum discrepancy of the resonance frequency below $4 \mathrm{~Hz}$.

\section{Conclusions}

The nodal DG method has been presented to solve the forced plate vibration problem. The upwind numerical flux of the 3-D linear elasticity equations has been presented in details using the Rankine-Hugoniot condition for the stress-velocity variables. Furthermore, the numerical aspects such as the point force excitation and the boundary conditions for the plates with S-S-S-S, S-S-S-F and S-SS-C boundary conditions have been implemented in the nodal DG methodology.

For the rectangular plate case, the nodal DG mobility shows an excellent agreement with the exact mobility of the first order shear deformation theory (FSDT). The agreement could be observed even at the location near to the point force excitation. On the other hand, a good agreement with the classical plate theory (CPT) happens solely at the first couple of resonance frequencies, with increased accuracy as the plate thickness is reduced.

For the L-shaped plate case, the nodal DG mobility shows a good agreement with the CPT and FSDT in the lower frequency range. However, in the higher frequency range, there are some disagreements due to significant frequency differences of the resonance and the anti-resonance frequencies. However, the nodal DG mobility has an excellent agreement with the mobility obtained by the finite element method of the 3-D linear elasticity equations. An acceleration of the nodal DG method by a parallel implementation will make this method highly useful for industrial applications, e.g., as presented for outdoor acoustics [21].

\section{Acknowledgments}

This research is supported by the ministry of finance of the Republic of Indonesia under framework of endowment fund for education (LPDP).

\section{Appendix}

In this appendix, the upwind flux $\mathbf{f}^{*}$ is given in details. The numerical flux can be written as

$$
\begin{aligned}
\mathbf{f}^{*}=\mathbf{A q}^{-}+ & {\left.\left[\begin{array}{l|l|l}
\mathbf{P}_{11} & \mathbf{P}_{12} & \mathbf{P}_{13} \\
\hline \mathbf{P}_{21} & \mathbf{P}_{22} & \mathbf{P}_{23} \\
\hline \mathbf{P}_{31} & \mathbf{P}_{32} & \mathbf{P}_{33}
\end{array}\right]-\left[\begin{array}{l|l|l}
\mathbf{S}_{11} & \mathbf{S}_{12} & \mathbf{S}_{13} \\
\hline \mathbf{S}_{21} & \mathbf{S}_{22} & \mathbf{S}_{23} \\
\hline \mathbf{S}_{31} & \mathbf{S}_{32} & \mathbf{S}_{33}
\end{array}\right]\right] } \\
& \cdot[[\mathbf{q}]]
\end{aligned}
$$

where $[[\mathbf{q}]]=\mathbf{q}^{+}-\mathbf{q}^{-}$. The components of the matrix are given below,

$$
\begin{aligned}
& \mathbf{P}_{11}=-\frac{c_{p}}{2}\left[\begin{array}{ccc}
n_{x}^{2} & n_{x} n_{y} & n_{x} n_{z} \\
n_{x} n_{y} & n_{y}^{2} & n_{y} n_{z} \\
n_{x} n_{z} & n_{y} n_{z} & n_{z}^{2}
\end{array}\right] \\
& \mathbf{P}_{21}=-\frac{1}{2}\left[\begin{array}{l}
n_{x}\left(\lambda+2 \mu n_{x}^{2}\right) \\
n_{x}\left(\lambda+2 \mu n_{y}^{2}\right) \\
n_{x}\left(\lambda+2 \mu n_{z}^{2}\right)
\end{array}\right] \\
& \left.\begin{array}{lll} 
& n_{y}\left(\lambda+2 \mu n_{x}^{2}\right) & n_{z}\left(\lambda+2 \mu n_{x}^{2}\right) \\
\cdots & n_{y}\left(\lambda+2 \mu n_{y}^{2}\right) & n_{z}\left(\lambda+2 \mu n_{y}^{2}\right) \\
& n_{y}\left(\lambda+2 \mu n_{z}^{2}\right) & n_{z}\left(\lambda+2 \mu n_{z}^{2}\right)
\end{array}\right]
\end{aligned}
$$$$
\mathbf{P}_{31}=-\mu\left[\begin{array}{ccc}
n_{x}^{2} n_{z} & n_{x} n_{y} n_{z} & n_{z}^{2} n_{x} \\
n_{x} n_{y} n_{z} & n_{y}^{2} n_{z} & n_{z}^{2} n_{y} \\
n_{x}^{2} n_{y} & n_{y}^{2} n_{x} & n_{x} n_{y} n_{z}
\end{array}\right]
$$$$
\mathbf{P}_{12}=-\frac{1}{2 \rho}\left[\begin{array}{ccc}
n_{x}^{3} & n_{y}^{2} n_{x} & n_{z}^{2} n_{x} \\
n_{x}^{2} n_{y} & n_{y}^{3} & n_{z}^{2} n_{y} \\
n_{x}^{2} n_{z} & n_{y}^{2} n_{z} & n_{z}^{3}
\end{array}\right]
$$$$
\mathbf{P}_{22}=-\frac{1}{2 Z_{p}}\left[\begin{array}{l}
n_{x}^{2}\left(\lambda+2 \mu n_{x}^{2}\right) \\
n_{x}^{2}\left(\lambda+2 \mu n_{y}^{2}\right) \\
n_{x}^{2}\left(\lambda+2 \mu n_{z}^{2}\right)
\end{array}\right]
$$$$
\left.\begin{array}{lll} 
& n_{y}^{2}\left(\lambda+2 \mu n_{x}^{2}\right) & n_{z}^{2}\left(\lambda+2 \mu n_{x}^{2}\right) \\
\cdots & n_{y}^{2}\left(\lambda+2 \mu n_{y}^{2}\right) & n_{z}^{2}\left(\lambda+2 \mu n_{y}^{2}\right) \\
& n_{y}^{2}\left(\lambda+2 \mu n_{y}^{2}\right) & n_{z}^{2}\left(\lambda+2 \mu n_{z}^{2}\right)
\end{array}\right]
$$$$
\mathbf{P}_{32}=-\frac{\mu}{Z_{p}}\left[\begin{array}{ccc}
n_{x}^{3} n_{z} & n_{y}^{2} n_{x} n_{z} & n_{z}^{3} n_{x} \\
n_{x}^{2} n_{y} n_{z} & n_{y}^{3} n_{z} & n_{z}^{3} n_{y} \\
n_{x}^{3} n_{y} & n_{y}^{3} n_{x} & n_{z}^{2} n_{x} n_{y}
\end{array}\right]
$$$$
\mathbf{P}_{13}=-\frac{1}{\rho}\left[\begin{array}{ccc}
n_{x}^{2} n_{z} & n_{x} n_{y} n_{z} & n_{x}^{2} n_{y} \\
n_{x} n_{y} n_{z} & n_{y}^{2} n_{z} & n_{y}^{2} n_{x} \\
n_{z}^{2} n_{x} & n_{z}^{2} n_{y} & n_{x} n_{y} n_{z}
\end{array}\right]
$$

$$
\begin{aligned}
\mathbf{P}_{23}=-\frac{1}{Z_{p}}\left[\begin{array}{l}
n_{x} n_{z}\left(\lambda+2 \mu n_{x}^{2}\right) \\
n_{x} n_{z}\left(\lambda+2 \mu n_{y}^{2}\right) \\
n_{x} n_{z}\left(\lambda+2 \mu n_{z}^{2}\right)
\end{array}\right. & \cdots \\
n_{y} n_{z}\left(\lambda+2 \mu n_{x}^{2}\right) & n_{x} n_{y}\left(\lambda+2 \mu n_{x}^{2}\right) \\
& \left.\begin{array}{lll}
n_{y} n_{z}\left(\lambda+2 \mu n_{y}^{2}\right) & n_{x} n_{y}\left(\lambda+2 \mu n_{y}^{2}\right) \\
& n_{y} n_{z}\left(\lambda+2 \mu n_{y}^{2}\right) & n_{x} n_{y}\left(\lambda+2 \mu n_{z}^{2}\right)
\end{array}\right]
\end{aligned}
$$

$$
\mathbf{P}_{33}=-\frac{2 \mu}{Z_{p}}\left[\begin{array}{ccc}
n_{x}^{2} n_{z}^{2} & n_{z}^{2} n_{x} n_{y} & n_{x}^{2} n_{y} n_{z} \\
n_{z}^{2} n_{x} n_{y} & n_{y}^{2} n_{z}^{2} & n_{y}^{2} n_{x} n_{z} \\
n_{x}^{2} n_{y} n_{z} & n_{y}^{2} n_{x} n_{z} & n_{x}^{2} n_{y}^{2}
\end{array}\right]
$$


$\mathbf{S}_{11}=-\frac{c_{s}}{2}\left[\begin{array}{ccc}n_{x}^{2}-1 & n_{x} n_{y} & n_{x} n_{z} \\ n_{x} n_{y} & n_{y}^{2}-1 & n_{y} n_{z} \\ n_{x} n_{z} & n_{y} n_{z} & n_{z}^{2}-1\end{array}\right]$

$\mathbf{S}_{21}=-\mu\left[\begin{array}{ccc}n_{x}\left(n_{x}^{2}-1\right) & n_{x}^{2} n_{y} & n_{x}^{2} n_{z} \\ n_{y}^{2} n_{x} & n_{y}\left(n_{y}^{2}-1\right) & n_{y}^{2} n_{z} \\ n_{z}^{2} n_{x} & n_{z}^{2} n_{y} & n_{z}\left(n_{z}^{2}-1\right)\end{array}\right]$

$$
\begin{aligned}
& \mathbf{S}_{31}=-\frac{\mu}{2}\left[\begin{array}{c}
n_{z}\left(2 n_{x}^{2}-1\right) \\
2 n_{x} n_{y} n_{z} \\
-n_{y}\left(2 n_{x}^{2}-1\right)
\end{array} \ldots\right.
\end{aligned}
$$

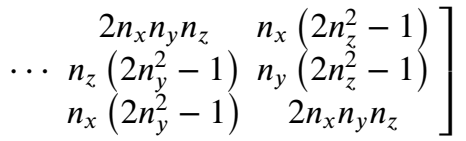$$
\mathbf{S}_{12}=-\frac{1}{2 \rho}\left[\begin{array}{ccc}
n_{x}\left(n_{x}^{2}-1\right) & n_{y}^{2} n_{x} & n_{z}^{2} n_{x} \\
n_{x}^{2} n_{y} & n_{y}\left(n_{y}^{2}-1\right) & n_{z}^{2} n_{y} \\
n_{x}^{2} n_{z} & n_{y}^{2} n_{z} & n_{z}\left(n_{z}^{2}-1\right)
\end{array}\right]
$$$$
\mathbf{S}_{22}=-c_{s}\left[\begin{array}{ccc}
n_{x}^{2}\left(n_{x}^{2}-1\right) & n_{x}^{2} n_{y}^{2} & n_{x}^{2} n_{z}^{2} \\
n_{x}^{2} n_{y}^{2} & n_{y}^{2}\left(n_{y}^{2}-1\right) & n_{y}^{2} n_{z}^{2} \\
n_{x}^{2} n_{z}^{2} & n_{y}^{2} n_{z}^{2} & n_{z}^{2}\left(n_{z}^{2}-1\right)
\end{array}\right]
$$

$$
\begin{aligned}
& \mathbf{S}_{32}=-\frac{c_{s}}{2}\left[\begin{array}{c}
n_{x} n_{z}\left(2 n_{x}^{2}-1\right) \\
2 n_{x}^{2} n_{y} n_{z} \\
n_{x} n_{y}\left(2 n_{x}^{2}-1\right)
\end{array} \ldots\right.
\end{aligned}
$$

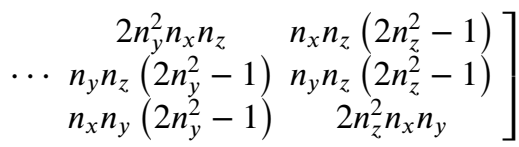

$$
\begin{aligned}
& \mathbf{S}_{13}=-\frac{1}{2 \rho}\left[\begin{array}{c}
n_{z}\left(2 n_{x}^{2}-1\right) \\
2 n_{x} n_{y} n_{z} \\
n_{x}\left(2 n_{z}^{2}-1\right)
\end{array} \ldots\right. \\
& \left.\begin{array}{cc}
2 n_{x} n_{y} n_{z} & n_{y}\left(2 n_{x}^{2}-1\right) \\
\cdots & n_{z}\left(2 n_{y}^{2}-1\right) \\
n_{y}\left(2 n_{z}^{2}-1\right) & n_{x}\left(2 n_{y}^{2}-1\right) \\
& 2 n_{x} n_{z} n_{y}
\end{array}\right]
\end{aligned}
$$

$$
\begin{aligned}
& \mathbf{S}_{23}=-c_{s}\left[\begin{array}{c}
n_{x} n_{z}\left(2 n_{x}^{2}-1\right) \\
2 n_{y}^{2} n_{x} n_{z} \\
n_{x} n_{z}\left(2 n_{z}^{2}-1\right)
\end{array} \ldots\right.
\end{aligned}
$$

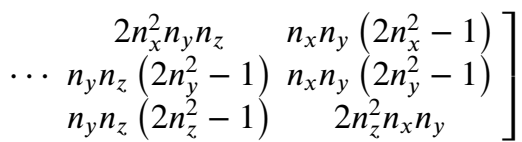

$$
\begin{aligned}
& \mathbf{S}_{33}=-\frac{c_{s}}{2}\left[\begin{array}{l}
4 n_{x}^{2} n_{z}^{2}+n_{y}^{2}-1 \\
n_{x} n_{y}\left(4 n_{z}^{2}-1\right) \\
n_{y} n_{z}\left(4 n_{x}^{2}-1\right)
\end{array}\right] \\
& \left.\begin{array}{lll} 
& n_{x} n_{y}\left(4 n_{z}^{2}-1\right) & n_{y} n_{z}\left(4 n_{x}^{2}-1\right) \\
\cdots & 4 n_{y}^{2} n_{z}^{2}+n_{x}^{2}-1 & n_{x} n_{z}\left(4 n_{y}^{2}-1\right) \\
& n_{x} n_{z}\left(4 n_{y}^{2}-1\right) & 4 n_{x}^{2} n_{y}^{2}+n_{z}^{2}-1
\end{array}\right],
\end{aligned}
$$

where $Z_{p}=\rho c_{p}$, notice that $\mathbf{P}$ components are the contributions of the longitudinal wave, and $\mathbf{S}$ components are the contributions of the shear wave.

\section{References}

[1] J. Brunskog: Energy based prediction models for building acoustics. Proceedings of the joint BalticNordic Acoustics Meeting, 2012.

[2] W. Soedel: Vibrations of shells and plates. Third edition. Marcel Dekker Inc., New York, 2004.

[3] A. Bermúdez, P.Gamallo, L. Hervella-Nieto, R. Rodríguez , D. Santamarina: Fluid-structure acoustic interaction. In: Computational Acoustics of Noise Propagation in Fluids - Finite and Boundary Element Methods. S. Marburg, B. Nolte (eds), Springer, Berlin, 2008.

[4] J. Virieux: P-SV wave propagation in heterogeneous media: velocity-stress finite-difference method. Geophysics 52(4) (1986) 889-901.

[5] M. Dumbser, M. Käser: An arbitrary high-order discontinuous Galerkin method for elastic waves on unstructured meshes - -II. The three-dimensional isotropic case. Geophysical Journal International 167(1) (2006) 319-336.

[6] L. C. Wilcox, G. Stadler, C.Burstedde, O. Ghattas: A highorder discontinuous Galerkin method for wave propagation through coupled elastic-acoustic media. Journal of Computational Physics 229 (2010) 9373-9396.

[7] M. Toyoda, D. Takahashi: Prediction for architectural structure-borne sound by the finite-difference time-domain method. Acoustical Science and Technology 30(4) (2009) 265-276.

[8] L. Xuan, G. Jin, J. Gong, W. Zhang, P. Ming: Time domain finite volume method for three-dimensional structuralacoustic coupling analysis. Applied Acoustics 76 (2014) 138-149.

[9] A. Bermúdez, L. Hervella-Nieto, R. Rodríguez: Finite element computation of three-dimensional elastoacoustic vibrations. Journal of Sound and Vibration 219(2) (1999) 279-306.

[10] J. S. Hesthaven, T. Warburton: Nodal discontinuous Galerkin methods: Algorithms, Analysis, and Applications. First edition, Springer, New York, 2007.

[11] R. P. Muñoz, M. Hornikx: Hybrid Fourier pseudospectral/discontinuous Galerkin time-domain method for wave propagation. Journal of Computational Physics 348 (2017) 416-432.

[12] R. J. LeVeque: Finite volume methods for hyperbolic problems. Cambridge University Press, Cambridge, 2002.

[13] T. Toulorge: Efficient Runge-Kutta discontinuous Galerkin methods applied to aero-acoustics, Ph.D dissertation, KU Leuven, Belgium, 2012.

[14] I. Sihar, M. Hornikx, Pranowo: Time-domain structural vibration simulations by solving the linear elasticity equations with the discontinuous Galerkin method. Proceedings of ICSV-24, 2017.

[15] C. Y. Wang, C. M. Wang: Structural vibration. CRC Press, Florida, 2014

[16] S. Srinivas, C. V. J. Rao, A. K. Rao: An exact analysis for vibration of simply-supported homogeneous and laminated thick rectangular plates. Journal of Sound and Vibration 12(2) (1970) 187-199.

[17] J. N. Reddy: Theory and analysis of elastic plates and shells. Second edition, CRC Press, Florida, 2007. 
[18] D. J. Gorman: Free vibration analysis of rectangular plates. Elsevier, Amsterdam, 1982.

[19] S. H. Hashemi, M. Arsanjani: Exact characteristic equations for some of classical boundary conditions of vibrating moderately thick rectangular plates. International Journal of Solids and Structures 42(3-4) (2005) 819-853.
[20] N. J. Kessissoglou: Power transmission in L-shaped plates including flexural and in-plane vibration. The Journal of the Acoustical Society of America 115(3) (2004) 1157-1169.

[21] S. Schoeder, W. A. Wall, M. Kronbichler: ExWave: A high performance discontinuous Galerkin solver for the acoustic wave equation. SoftwareX 9 (2019) 49-54. 